\title{
Uniqueness theorem on meromorphic functions and their difference operators
}

\author{
Dan Liu ${ }^{1}$, Bingmao Deng ${ }^{1}$ and Mingliang Fang ${ }^{1 *}$
}

${ }^{*}$ Correspondence:

mlfang@scau.edu.cn

${ }^{1}$ Institute of Applied Mathematics, South China Agricultural University,

Guangzhou, China

\begin{abstract}
In this paper, we study the uniqueness problems of meromorphic functions and their difference operators. Our main result is a difference analogue of a result of Jank-Mues-Volkmann, which is concerned with the uniqueness of an entire function sharing one finite value with its derivatives. Some recent papers studied the case of entire functions of finite order sharing a periodic small function to $f$. We consider the case of meromorphic functions of finite order sharing a polynomial, which is a more popular case. Examples are provided for our results.
\end{abstract}

MSC: Primary 30D35; secondary 39B32

Keywords: Uniqueness; Meromorphic functions; Difference operators

\section{Introduction and main results}

Let $\mathbb{C}$ denote the complex plane and $f$ a meromorphic function in the whole complex plane $\mathbb{C}$. We use the following standard notations of the Nevanlinna value distribution theory $($ see $[11,15,16])$ :

$$
m(r, f), N(r, f), \bar{N}(r, f), T(r, f), \rho(f), S(r, f), \ldots
$$

where

$$
\begin{aligned}
& m(r, f)=\frac{1}{2 \pi} \int_{0}^{2 \pi} \log ^{+} \mid f\left(r e^{i \theta} \mid\right) d \theta, \\
& N(r, f)=\int_{0}^{r} \frac{n(t, f)-n(0, f)}{t} d t+n(0, f) \log r, \\
& \bar{N}(r, f)=\int_{0}^{r} \frac{\bar{n}(t, f)-\bar{n}(0, f)}{t} d t+\bar{n}(0, f) \log r, \\
& T(r, f)=m(r, f)+N(r, f) .
\end{aligned}
$$

Among them, $m(r, f)$ is the average of the positive logarithm of $|f(z)|$ on the circle $|z|=$ $r, N(r, f)$ is called the counting function of poles of $f$, and $\bar{N}(r, f)$ is called the reduced counting function of poles of $f . T(r, f)$ is called the characteristic function of $f$, and it plays a cardinal role in the whole theory of meromorphic functions.

(c) The Author(s) 2018. This article is distributed under the terms of the Creative Commons Attribution 4.0 International License (http://creativecommons.org/licenses/by/4.0/), which permits unrestricted use, distribution, and reproduction in any medium, provided you give appropriate credit to the original author(s) and the source, provide a link to the Creative Commons license, and indicate if changes were made. 
The order of growth of $f$ is denoted by $\rho(f)$ as follows:

$$
\rho(f)=\varlimsup_{r \rightarrow \infty} \frac{\log ^{+} T(r, f)}{\log r} .
$$

If $\rho(f)<\infty$, then we say that $f$ is a meromorphic function of finite order.

For a meromorphic function $a$, if $T(r, a)=S(r, f)$, where $S(r, f)=o(T(r, f))$, as $r \rightarrow \infty$, possibly outside of an exceptional set of finite logarithmic measure, then we say that $a$ is a small function of $f$. We use $S(f)$ to denote the set of the small functions of $f$.

For a meromorphic function $f(z)$, we define its shift by $f_{c}(z)=f(z+c)$ and its difference operators by

$$
\Delta_{c} f(z)=f(z+c)-f(z), \quad \Delta_{c}^{n} f(z)=\Delta_{c}^{n-1}\left(\Delta_{c} f(z)\right), \quad n \in \mathbb{N}, n \geq 2 .
$$

Let $f$ and $g$ be two meromorphic functions, and let $P(z)$ be a polynomial. We say that $f$ and $g$ share $P(z)$ CM, provided that $f(z)-P(z)$ and $g(z)-P(z)$ have the same zeros counting multiplicities.

In 1986, Jank et al. proved the following.

Theorem 1 ([12]) Let $f$ be a nonconstant meromorphic function, and let a be a nonzero finite complex number. If $f, f^{\prime}$, and $f^{\prime \prime}$ share a $C M$, then $f \equiv f^{\prime}$.

Later on, the uniqueness theorem of entire functions sharing a constant with its $k$ th and $(k+1)$ th derivatives was proved by Li and Yang in 2001 .

Theorem 2 ([14]) Letf be a nonconstant entire function; let a be a finite nonzero constant, and let $k$ be a positive integer. If $f, f^{(k)}, f^{(k+1)}$ share the value a $C M$, then $f \equiv f^{\prime}$.

In 2004, Chang and Fang extended Theorem 2 to the case of sharing small functions.

Theorem 3 ([2]) Let $f$ be a nonconstant entire function; let $a \not \equiv 0$ be a small function related to $f$; and let $k \geq 2$ be a positive integer. If $f, f^{(k)}$, and $f^{(k+1)}$ share a $C M$, then $f \equiv f^{\prime}$.

In recent years, the value distribution of meromorphic functions with respect to difference has become a subject of some interests (see [1, 3-5, 7-10, 13]). Chen et al. [3] proved a similar result analogue of Theorem 1 concerning difference. In 2015, Latreuch et al. studied the case of entire function with its difference analogue of Theorem 2 and Theorem 3 and proved the following.

Theorem 4 ([13]) Let $f$ be a nonconstant entire function of finite order, and let a $(z)(\not \equiv 0)$ $\in S(f)$ be a periodic entire function with period c. If $f, \Delta_{c}^{n} f$, and $\Delta_{c}^{n+1} f(n \geq 1)$ share a $(z)$ $C M$, then $\Delta_{c}^{n+1} f \equiv \Delta_{c}^{n} f$.

Theorem 5 ([13]) Letf be a nonconstant entire function of finite order. If $f, \Delta_{c}^{n} f$, and $\Delta_{c}^{n+1} f$ $(n \geq 1)$ share $0 C M$, then $\Delta_{c}^{n+1} f \equiv C \Delta_{c}^{n} f$, where $C$ is a nonzero constant.

Remark 1 There are examples to show that the conclusion $\Delta_{c}^{n} f \equiv \Delta_{c}^{n+1} f$ in Theorem 4 cannot be replaced by $f \equiv \Delta_{c} f$, and the condition $a(z) \not \equiv 0$ is necessary. 
In 2016, El Farissi et al. successfully proved the relation between $f$ and $\Delta_{f} f$ under the condition that $a(z)$ is a periodic function.

Theorem 6 ([8]) Let $f(z)$ be a nonconstant entire function of finite order such that $\Delta_{c}^{n} f(z) \not \equiv$ 0 , and let $a(z)(\not \equiv 0) \in S(f)$ be a periodic entire function with period c. If $f, \Delta_{c}^{n} f$, and $\Delta_{c}^{n+1} f$ $(n \geq 1)$ share $a(z) C M$, then $\Delta_{c} f(z) \equiv f(z)$.

For other related results, see Latreuch, El Farissi, Belädi [13], El Farissi, Latreuch, Asiri [7], El Farissi et al. [8].

By Theorems 4 and 6, we naturally have the following problems:

(i) Can we get rid of the condition that $a(z)$ is a periodic entire function with period $c$ in Theorem 6?

(ii) Since the case $n=1$ has been proved by Deng, Liu, and Fang [6] under the condition that $a(z)$ is a polynomial, can we still deduce that $f \equiv \Delta_{c} f$ for $n \geq 2$ ?

Theorem 7 ([6]) Let $f$ be a nonconstant meromorphic function of finite order, and let $p(z)$ $(\not \equiv 0)$ be a polynomial. If $f, \Delta_{c} f$, and $\Delta_{c}^{2} f$ share $\infty$ and $p(z) C M$, then either $f \equiv \Delta_{c} f$ or $f(z)=e^{A z+B}+b$, where $p(z) \not \equiv b(\neq 0)$, and $A \neq 0$ satisfying $e^{A c}=1$.

In this paper, we study these problems and give an affirmative answer to them.

Theorem 8 Let $f$ be a nonconstant meromorphic function of finite order, let $n \in \mathbb{N}^{+}$be a positive integer, and let $P(z)(\not \equiv 0)$ be a polynomial. If $f, \Delta_{c}^{n} f$, and $\Delta_{c}^{n+1} f$ share $\infty$ and $P(z)$ $C M$, then one of the following three cases must occur:

(i) $f \equiv \Delta_{c} f$;

(ii) $f=\left(1-e^{\alpha(z)}\right) P(z)$, where $e^{\alpha(z)}$ is a periodic function of period $c$ with $\operatorname{deg} \alpha=1$, and $P(z)$ is a polynomial of degree less than $n$;

(iii) $f(z)=A P(z)$, where $A(\neq 0,1)$ is a constant, and $P(z)$ is a polynomial of degree less than $n$.

Corollary 1 Let $f$ be a transcendental entire function of finite order, and let $P(z)(\not \equiv 0)$ be a polynomial. If $f, \Delta_{c}^{n} f$, and $\Delta_{c}^{n+1} f$ share $P(z) C M$, then either $f \equiv \Delta_{c} f$, or $f=\left(1-e^{\alpha(z)}\right) P(z)$, where $e^{\alpha(z)}$ is a periodic function of period $c$ with $\operatorname{deg} \alpha=1$, and $P(z)$ is a polynomial with $\operatorname{deg} P \leq n$.

Problem 1 In this paper, we study the uniqueness of meromorphic functions of finite order sharing a polynomial with its differences. We have the following question: What can we say if sharing a meromorphic function? What is more, does this result still hold for meromorphic functions of arbitrary order?

In the following, we give three cases to show that all these cases may occur.

Example 1 Let $A, c$ be two nonzero finite complex numbers satisfying $e^{A c}=2$, and let $f(z)=e^{A z}$. Then $f(z)=\Delta_{c}^{n} f=\Delta_{c}^{n+1} f=e^{A z}(n \geq 1)$, and for any polynomial $P(z)$, we have $f$, $\Delta_{c}^{n} f$, and $\Delta_{c}^{n+1} f$ share $\infty$ and $P(z) C M$. This example satisfies Case (i) of Theorem 8.

Example 2 Let $P(z)$ be a polynomial with $\operatorname{deg} P \leq n-1, f(z)=\left(e^{\pi i z}+1\right) P(z)$, and $c=2$. Then $\Delta_{c}^{j} f=\left(e^{\pi i z}+1\right) \Delta_{c}^{j} P$ for each $j \in \mathbb{N}^{+}$, it follows that $\Delta_{c}^{n} f \equiv \Delta_{c}^{n+1} f \equiv 0$ since $\Delta_{c}^{n} P \equiv$ 
$\Delta_{c}^{n+1} P \equiv 0$. Therefore, $f-P=e^{\pi i z} P, \Delta_{c}^{n} f-P=-P$, and $\Delta_{c}^{n+1} f-P=-P$ have the same zeros with the same multiplicities, and $f=\left(1+e^{\pi i z}\right) P(z)$, where $e^{\pi i z}$ is a periodic function of period $c=2$. This example shows that Case (ii) in Theorem 8 exists.

Example 3 Let $f(z)=2 z^{n-1}, P(z)=z^{n-1}$. It is obvious that $f(z)-P(z)=z^{n-1}, \Delta_{c}^{n} f(z)-P(z)=$ $\Delta_{c}^{n+1} f(z)-P(z)=-z^{n-1}$ have the same zeros. Thus $f(z), \Delta_{c}^{n} f(z)$, and $\Delta_{c}^{n+1} f(z)$ share $P(z)$, $\infty$ CM. This example shows that Case (iii) in Theorem 8 exists.

\section{Some lemmas}

Lemma $1([5,9])$ Let $f(z)$ be a meromorphic function of finite order, and let c be a nonzero complex constant. Then

$$
T(r, f(z+c))=T(r, f)+S(r, f) .
$$

Lemma $2([9,10])$ Let $c \in \mathbb{C}, k$ be a positive integer, and let $f(z)$ be a meromorphic function of finite order. Then

$$
m\left(r, \frac{\Delta_{c}^{k} f(z)}{f(z)}\right)=S(r, f)
$$

Lemma 3 ([16]) Suppose that $f(z)$ is a meromorphic function in the complex plane and $P(z)=a_{0} f^{n}+a_{1} f^{n-1}+\cdots+a_{n}$, where $a_{0}(\not \equiv 0), a_{1}, \ldots, a_{n}$ are small functions of $f(z)$. Then

$$
T(r, P(f))=n T(r, f)+S(r, f)
$$

Lemma $4([15,16])$ Suppose that $f_{i}(z)(i=1,2, \ldots, n)$ are meromorphic functions and $g_{i}(z)$ $(i=1,2, \ldots, n), n \geq 2$ are entire functions satisfying

(i) $\sum_{i=1}^{n} f_{i}(z) e^{g_{i}(z)} \equiv 0$,

(ii) the orders of $f_{i}$ are less than those of $e^{g_{k}-g_{l}}$ for $1 \leq i \leq n, 1 \leq k<l \leq n$.

Then $f_{i}(z) \equiv 0(i=1,2, \ldots, n)$.

Lemma $5([15,16])$ Suppose that $f_{1}(z), f_{2}(z), \ldots, f_{n}(z)(n \geq 3)$ are meromorphic functions which are not constants except for $f_{n}(z)$. Furthermore, let $\sum_{j=1}^{n} f_{j}(z)=1$. If $f_{n}(z) \not \equiv 0$ and

$$
\sum_{j=1}^{n} N\left(r, \frac{1}{f_{j}}\right)+(n-1) \sum_{j=1}^{n} \bar{N}\left(r, f_{j}\right)<(\lambda+o(1)) T\left(r, f_{k}\right)
$$

where $\lambda<1$ and $k=1,2, \ldots, n-1$, then $f_{n} \equiv 1$.

Lemma 6 ([16]) Suppose that $f(z)$ and $g(z)$ are two nonconstant meromorphic functions in the complex plane with $\rho(f)$ and $\rho(g)$ as their orders, respectively. Then

$$
\rho(f \cdot g) \leq \max \{\rho(f), \rho(g)\}
$$

and

$$
\rho(f+g) \leq \max \{\rho(f), \rho(g)\}
$$


Using the ideas of Chang and Fang [2] and El Farissi et al. [7, 8], we prove the following lemma.

Lemma 7 Let $f$ be a nonconstant meromorphic function of finite order, and let $P(z)(\equiv 0)$ be a polynomial, $n$ be a positive integer. Suppose that

$$
\frac{\Delta_{c}^{n} f(z)-P(z)}{f(z)-P(z)}=e^{\alpha(z)}, \quad \frac{\Delta_{c}^{n+1} f(z)-P(z)}{f(z)-P(z)}=e^{\beta(z)},
$$

where $\alpha(z)$ and $\beta(z)$ are two polynomials. If

$$
T\left(r, e^{\alpha}\right)+T\left(r, e^{\beta}\right)=S(r, f)
$$

then either $f(z)=A P(z)$, where $\alpha, \beta, A=\frac{e^{\alpha}-1}{e^{\alpha}}$ are constants and $P(z)$ is a polynomial with degree less than $n$, or $f \equiv \Delta_{c} f$.

Proof Two cases will be discussed in the following.

Case 1. $f(z)$ is a rational function.

Firstly, we claim that $f(z)$ cannot be a non-polynomial rational function. Otherwise, suppose that $f(z)=\frac{a(z)}{b(z)}$, where $a(z)$ and $b(z)$ are two co-prime polynomials. By equation (2.1), we can get that $f, \Delta_{c}^{n} f$, and $\Delta_{c}^{n+1} f$ share $\infty \mathrm{CM}$. Then $\Delta_{c}^{n} f$ and $\Delta_{c}^{n+1} f$ are rational functions, too. Suppose that $\Delta_{c}^{n} f=\frac{g(z)}{b(z)}$, where $g(z)$ and $b(z)$ are two co-prime polynomials. We claim that $b(z)$ is a constant. Otherwise, suppose that there exists $z_{0}$ such that $b\left(z_{0}+c\right)=0$. Since $\Delta_{c}^{n} f$ and $\Delta_{c}^{n+1} f$ share $\infty \mathrm{CM}$, and

$$
\Delta_{c}^{n+1} f=\Delta_{c}^{n} f(z+c)-\Delta_{c}^{n} f(z)=\frac{g(z+c)}{b(z+c)}-\frac{g(z)}{b(z)}=\frac{g(z+c) b(z)-g(z) b(z+c)}{b(z) b(z+c)},
$$

then all zeros of $b(z+c)$ must be the zeros of $b(z)$. Otherwise, if there exists $z_{1}$ such that $b\left(z_{1}+c\right)=0$ but $b\left(z_{1}\right) \neq 0$, then $z_{1}$ is one pole of $\Delta_{c}^{n+1} f$ but not the pole of $\Delta_{c}^{n} f$, a contradiction. So we get

$$
\begin{aligned}
& b\left(z_{0}+c\right)=0 \quad \Rightarrow \quad b\left(z_{0}\right)=0 \quad \Rightarrow \quad b\left(z_{0}-c\right)=0 \quad \Rightarrow \quad \cdots \quad \Rightarrow \quad b\left(z_{0}-k c\right)=0, \\
& k \in \mathbb{N}^{+}
\end{aligned}
$$

which implies that $b(z)$ has infinitely many zeros, a contradiction.

Therefore, $f(z)$ is a nonconstant polynomial.

Without loss of generality, we assume that

$$
\begin{aligned}
& f(z)=a_{l} z^{l}+a_{l-1} z^{l-1}+\cdots+a_{1} z+a_{0} \quad\left(a_{l} \neq 0\right), \\
& P(z)=b_{m} z^{m}+b_{m-1} z^{m-1}+\cdots+b_{1} z+b_{0} \quad\left(b_{m} \neq 0\right) .
\end{aligned}
$$

By equation (2.1), it is easy to prove that $\alpha(z), \beta(z)$ are two constants, then

$$
\left\{\begin{array}{l}
\Delta_{c}^{n} f-P(z)=e^{\alpha} f-e^{\alpha} P(z), \\
\Delta_{c}^{n+1} f-P(z)=e^{\beta} f-e^{\beta} P(z) .
\end{array}\right.
$$


If $e^{\alpha}=1\left(\right.$ or $\left.e^{\beta}=1\right)$, then $f \equiv \Delta_{c}^{n} f$ (or $f \equiv \Delta_{c}^{n+1} f$ ), which contradicts $\operatorname{deg} f>\operatorname{deg} \Delta_{c}^{n} f$ (or $\left.\operatorname{deg} f>\operatorname{deg} \Delta_{c}^{n+1} f\right)$. Thus $e^{\alpha}, e^{\beta} \neq 1$.

Comparing the degree of two equations, it follows that $\operatorname{deg} f=l \leq m=\operatorname{deg} P$.

If $\operatorname{deg} f=l<m=\operatorname{deg} P$, then $e^{\alpha}=e^{\beta}=1$, then $f \equiv \Delta_{c}^{n} f \equiv \Delta_{c}^{n+1} f$, which is a contradiction.

If $\operatorname{deg} f=l=m=\operatorname{deg} P$, comparing the coefficients of $z^{m}$ of both sides of equation (2.1), we immediately have

$$
a_{l} e^{\alpha}=\left(e^{\alpha}-1\right) b_{m}, \quad a_{l} e^{\beta}=\left(e^{\beta}-1\right) b_{m},
$$

it follows that $e^{\alpha}=e^{\beta}$, then we get $\Delta_{c}^{n} f \equiv \Delta_{c}^{n+1} f$.

Since $f(z)$ is a polynomial, then $\Delta_{c}^{n} f \equiv \Delta_{c}^{n+1} f \equiv 0$, which means $f(z)$ is a polynomial with degree less than $n$.

By equation (2.1), we have

$$
-P(z)=e^{\alpha} f(z)-e^{\alpha} P(z),
$$

then $f(z)=A P(z)$, where $A=\frac{e^{\alpha}-1}{e^{\alpha}}(\neq 0,1)$ is a constant, $P(z)$ is a polynomial with degree less than $n$.

Case 2. $f(z)$ is a transcendental meromorphic function, then $T(r, P)=S(r, f)$. By equation (2.1), we have

$$
\begin{aligned}
& \Delta_{c}^{n} f=e^{\alpha} f(z)-\left(e^{\alpha}-1\right) P(z) ; \\
& \Delta_{c}^{n+1} f=e^{\beta} f(z)-\left(e^{\beta}-1\right) P(z),
\end{aligned}
$$

then

$$
\begin{aligned}
\Delta_{c}^{n+1} f & =\Delta_{c}^{n} f(z+c)-\Delta_{c}^{n} f(z) \\
& =e^{\alpha(z+c)} f(z+c)-\left(e^{\alpha(z+c)}-1\right) P(z+c)-e^{\alpha(z)} f+\left(e^{\alpha}-1\right) P \\
& =e^{\beta(z)} f-\left(e^{\beta}-1\right) P .
\end{aligned}
$$

Therefore,

$$
\begin{aligned}
f_{c}(z) & =f(z+c) \\
& =\left(e^{\alpha-\alpha_{c}}+e^{\beta-\alpha_{c}}\right) f+\left(1-e^{-\alpha_{c}}\right) P_{c}-\left(e^{\alpha-\alpha_{c}}+e^{\beta-\alpha_{c}}-2 e^{-\alpha_{c}}\right) P \\
& =A(z) f(z)+B(z),
\end{aligned}
$$

where $A(z)=\left(e^{\alpha-\alpha_{c}}+e^{\beta-\alpha_{c}}\right), B(z)=\left(1-e^{-\alpha_{c}}\right) P_{c}-\left(e^{\alpha-\alpha_{c}}+e^{\beta-\alpha_{c}}-2 e^{-\alpha_{c}}\right) P$.

Then

$$
\begin{aligned}
f_{2 c}(z) & =f_{c}(z+c) \\
& =A(z+c) f(z+c)+B(z+c) \\
& =A(z+c)(A(z) f(z)+B(z))+B(z+c) \\
& =A(z+c) A(z) f(z)+A(z+c) B(z)+B(z+c) \\
& =A_{2}(z) f(z)+B_{2}(z),
\end{aligned}
$$


where $A_{2}(z)=A(z+c) A(z)=\left(e^{\alpha_{c}-\alpha_{2 c}}+e^{\beta_{c}-\alpha_{2 c}}\right)\left(e^{\alpha-\alpha_{c}}+e^{\beta-\alpha_{c}}\right)=e^{-\left(\alpha_{c}+\alpha_{2 c}\right)}\left(e^{\alpha_{c}}+e^{\beta_{c}}\right)\left(e^{\alpha}+e^{\beta}\right)$, and $B_{2}(z)=A(z+c) B(z)+B(z+c)$.

By mathematical induction, we can deduce that

$$
f_{i c}(z)=A_{i}(z) f(z)+B_{i}(z) \quad(i \geq 1)
$$

where $A_{i}(z)=\prod_{k=0}^{i-1} A_{k c}(z)=\prod_{k=1}^{i} e^{-\alpha_{k c}}\left(e^{\beta_{(k-1) c}}+e^{\alpha_{(k-1) c}}\right), B_{i}(z)$ is a meromorphic function depending on $P(z), e^{\alpha(z)}, e^{\beta(z)}$ and their differences.

By equation (2.5),

$$
\begin{aligned}
\Delta_{c}^{n} f & =\sum_{i=0}^{n} C_{n}^{i}(-1)^{n-i} f_{i c}(z) \\
& =(-1)^{n} f(z)+\sum_{i=1}^{n} C_{n}^{i}(-1)^{n-i}\left[\left(\prod_{k=1}^{i} e^{-\alpha_{k c}}\left(e^{\beta_{(k-1) c}}+e^{\alpha_{(k-1) c}}\right)\right) f(z)+B_{i}(z)\right] \\
& =e^{\alpha} f-\left(e^{\alpha}-1\right) P(z) .
\end{aligned}
$$

Then

$$
\begin{aligned}
& {\left[\sum_{i=1}^{n} C_{n}^{i}(-1)^{n-i}\left(\prod_{k=1}^{i} e^{-\alpha_{k c}}\left(e^{\beta_{(k-1) c}}+e^{\alpha_{(k-1) c}}\right)\right)+(-1)^{n}-e^{\alpha}\right] f(z)} \\
& \quad+\sum_{i=1}^{n} C_{n}^{i}(-1)^{n-i} B_{i}(z)+\left(e^{\alpha}-1\right) P(z)=0 .
\end{aligned}
$$

We rewrite (2.7) as follows:

$$
S(z) f(z)+T(z)=0,
$$

where $S(z)=\sum_{i=1}^{n} C_{n}^{i}(-1)^{n-i}\left(\prod_{k=1}^{i} e^{-\alpha_{k c}}\left(e^{\beta_{(k-1) c}}+e^{\alpha(k-1) c}\right)\right)+(-1)^{n}-e^{\alpha}$,

$$
T(z)=\sum_{i=1}^{n} C_{n}^{i}(-1)^{n-i} B_{i}(z)+\left(e^{\alpha}-1\right) P(z) .
$$

It is obvious that $S(z)$ and $T(z)$ are all small functions with respect to $f(z)$. If $S(z) \not \equiv 0$, we can deduce from equation (2.8) that

$$
T(r, f)=S\left(r,-\frac{T(z)}{S(z)}\right)=S(r, f)
$$

a contradiction.

Hence $S(z) \equiv 0$, that is,

$$
\begin{aligned}
& \sum_{i=1}^{n} C_{n}^{i}(-1)^{n-i}\left(\prod_{k=1}^{i} e^{-\alpha_{k c}}\left(e^{\beta_{(k-1) c}}+e^{\alpha_{(k-1) c}}\right)\right)+(-1)^{n}-e^{\alpha}=0, \\
& \sum_{i=1}^{n} C_{n}^{i}(-1)^{n-i}\left(\prod_{k=1}^{i} e^{-\alpha_{k c}}\left(e^{\beta_{(k-1) c}}+e^{\alpha_{(k-1) c}}\right)\right)=e^{\alpha}-(-1)^{n},
\end{aligned}
$$




$$
\begin{aligned}
& \sum_{i=1}^{n} C_{n}^{i}(-1)^{n-i} e^{-\sum_{k=1}^{i} \alpha_{k c}} e^{\sum_{k=1}^{i} \alpha_{(k-1) c}} \prod_{k=1}^{i}\left(1+e^{\beta_{(k-1) c}-\alpha_{(k-1) c}}\right) \\
& \quad=e^{\alpha}-(-1)^{n},
\end{aligned}
$$

and

$$
\sum_{i=1}^{n} C_{n}^{i}(-1)^{n-i} e^{\alpha-\alpha_{i c}} \prod_{k=0}^{i-1}\left(1+e^{\beta_{k c}-\alpha_{k c}}\right)=e^{\alpha}-(-1)^{n}
$$

Let $\gamma_{k c}(z)=\beta_{k c}(z)-\alpha_{k c}(z), k \in\{1,2, \ldots, n\}$, and $\gamma(z)=\beta(z)-\alpha(z)$ when $k=0$.

So equation (2.9) can be written as follows:

$$
\sum_{i=1}^{n} C_{n}^{i}(-1)^{n-i} e^{\alpha-\alpha_{i c}} \prod_{k=0}^{i-1}\left(1+e^{\gamma_{k c}}\right)=e^{\alpha}-(-1)^{n}
$$

For

$$
\begin{aligned}
\prod_{k=0}^{i-1}\left(1+e^{\gamma_{k c}}\right) \\
=\left(1+e^{\gamma}\right)\left(1+e^{\gamma_{c}}\right)\left(1+e^{\gamma_{2 c}}\right) \cdots\left(1+e^{\gamma_{(i-1) c}}\right) \\
=1+\left(e^{\gamma}+e^{\gamma_{c}}+\cdots+e^{\gamma_{(i-1) c}}\right)+\left(e^{\gamma+\gamma_{c}}+e^{\gamma+\gamma_{2 c}}+\cdots+e^{\gamma_{(i-2) c}+\gamma_{(i-1) c}}\right) \\
\quad+\cdots+e^{\gamma+\gamma_{c}+\cdots+\gamma_{(i-1) c}}
\end{aligned}
$$

Next, we discuss two cases.

Case 2.1. $\gamma(z)$ is a nonconstant polynomial.

Set $\gamma(z)=a_{m} z^{m}+a_{m-1} z^{m-1}+\cdots+a_{1} z+a_{0}$, and $a_{m} \neq 0$.

Since $\gamma(z)=\beta(z)-\alpha(z)$, we claim that $\operatorname{deg} \gamma(z) \geq \operatorname{deg} \alpha(z)$.

When $\operatorname{deg} \alpha \neq \operatorname{deg} \beta$, then $\operatorname{deg} \gamma=\max \{\operatorname{deg} \alpha, \operatorname{deg} \beta\}$;

When $\operatorname{deg} \alpha=\operatorname{deg} \beta$, then $\operatorname{deg} \gamma \leq \operatorname{deg} \alpha$.

If $\operatorname{deg} \gamma<\operatorname{deg} \alpha$, by equation (2.10), the order of the left-hand side

$$
\begin{aligned}
& \rho\left(C_{n}^{1}(-1)^{n-1} e^{\alpha-\alpha_{i c}}\left(1+e^{\gamma}\right)+C_{n}^{2}(-1)^{n-2} e^{\alpha-\alpha_{2 c}}\left(1+e^{\gamma}\right)\left(1+e^{\gamma_{c}}\right)\right. \\
& \left.\quad+\cdots+C_{n}^{n} e^{\alpha-\alpha_{n c}} \prod_{k=0}^{n-1}\left(1+e^{\gamma_{k c}}\right)\right) \\
& \quad \leq \operatorname{deg} \alpha-1,
\end{aligned}
$$

but the order of the right-hand side $\rho\left(e^{\alpha}-(-1)^{n}\right)=\operatorname{deg} \alpha$, a contradiction.

Thus $m=\operatorname{deg} \gamma \geq \operatorname{deg} \alpha$.

By equation (2.10), we have

$$
\alpha_{0}+\alpha_{1} e^{a_{m} z^{m}}+\alpha_{2} e^{2 a_{m} z^{m}}+\cdots+\alpha_{n} e^{n a_{m} z^{m}}=e^{\alpha},
$$


where $\alpha_{0}=(-1)^{n}+\sum_{i=1}^{n} C_{n}^{i}(-1)^{n-i} e^{\alpha-\alpha_{i c}}=e^{\alpha}\left[(-1)^{n}+\sum_{i=1}^{n} C_{n}^{i}(-1)^{n-i} e^{-\alpha_{i c}}\right]=e^{\alpha} \times$ $\sum_{i=0}^{n} C_{n}^{i}(-1)^{n-i} e^{-\alpha_{i c}}=e^{\alpha} \Delta_{c}^{n} e^{-\alpha}$, and $\alpha_{n}=e^{\alpha-\alpha_{n c}}$. We consider two subcases as follows.

Case 2.1.1. $\operatorname{deg} \alpha<m$.

It is obvious that $\alpha_{n} \neq \equiv 0$, by Lemma 1 and (2.11), we can get

$$
\operatorname{deg} \alpha=\rho\left(e^{\alpha}\right)=\rho\left(\alpha_{0}+\alpha_{1} e^{a_{m} z^{m}}+\alpha_{2} e^{2 a_{m} z^{m}}+\cdots+\alpha_{n} e^{n a_{m} z^{m}}\right)=m
$$

which is a contradiction.

Case 2.1.2. $\operatorname{deg} \alpha=m$.

Set $\alpha(z)=b_{m} z^{m}+\alpha^{*}(z)$, where $b_{m} \neq 0$, and $\operatorname{deg} \alpha^{*}(z) \leq m-1$. We claim that there exists at least $j \in\{1,2, \ldots, n\}$ such that $b_{m}=j a_{m}$.

Otherwise, $d \neq i a_{m}, i \in\{1,2, \ldots, n\}$, then by (2.11),

$$
\alpha_{1} e^{a_{m} z^{m}}+\alpha_{2} e^{2 a_{m} z^{m}}+\cdots+\alpha_{n} e^{n a_{m} z^{m}}-e^{b_{m} z^{m}} e^{\alpha^{*}(z)}=-\alpha_{0}
$$

Then by Lemma 4, we deduce that

$$
\alpha_{i}=e^{\alpha^{*}(z)}=0 \quad(i=1,2, \ldots, n)
$$

a contradiction.

Thus there exists $j \in\{1,2, \ldots, n\}$ such that $b_{m}=j a_{m}$. Without loss of generality, we assume that $b_{m}=n a_{m}$, then

$$
\alpha_{1} e^{a_{m} z^{m}}+\alpha_{2} e^{2 a_{m} z^{m}}+\cdots+\left(\alpha_{n}-e^{\alpha^{*}(z)}\right) e^{n a_{m} z^{m}}+\alpha_{0}=0
$$

By Lemma 4, we have $\alpha_{0} \equiv 0$, that is, $e^{\alpha} \Delta_{c}^{n} e^{-\alpha} \equiv 0$, then

$$
\Delta_{c}^{n} e^{-\alpha}=\sum_{i=0}^{n} C_{n}^{i}(-1)^{n-i} e^{-\alpha_{i c}} \equiv 0 .
$$

We claim that $\operatorname{deg} \alpha=\operatorname{deg} \gamma=m=1$. Otherwise, suppose that $\operatorname{deg} \alpha=\operatorname{deg} \gamma=m>1$. Let $\alpha(z)=b_{m} z^{m}+b_{m-1} z^{m-1}+\cdots+b_{1} z+b_{0}$, and $b_{m} \neq 0$.

Then

$$
\begin{aligned}
\alpha(z+j c) & =b_{m}(z+j c)^{m}+b_{m-1}(z+j c)^{m-1}+\cdots+b_{1}(z+j c)+b_{0} \\
& =b_{m} z^{m}+\left(b_{m-1}+m b_{m} j c\right) z^{m-1}+\mu_{j}(z)
\end{aligned}
$$

where $\mu_{j}(z)$ is a polynomial with $\operatorname{deg} \mu_{j}<m-1$.

Hence equation (2.12) can be written as follows:

$$
\begin{aligned}
& e^{-\mu_{n}(z)} e^{-b_{m} z^{m}-\left(b_{m-1}+m b_{m} n c\right) z^{m-1}}-C_{n}^{1} e^{-\mu_{n-1}(z)} e^{-b_{m} z^{m}-\left(b_{m-1}+m b_{m}(n-1) c\right) z^{m-1}} \\
& +\cdots+(-1)^{n} e^{-\mu_{0}(z)} e^{-b_{m} z^{m}-b_{m-1} z^{m-1}}=0 .
\end{aligned}
$$

It is obvious that

$$
\rho\left(e^{-b_{m} z^{m}-\left(b_{m-1}+m b_{m} k c\right) z^{m-1}+b_{m} z^{m}+\left(b_{m-1}+m b_{m} l c\right) z^{m-1}}\right)=\rho\left(e^{m b_{m}(l-k) z^{m-1}}\right)=m-1
$$

for any $0 \leq l<k \leq n$. 
Since $\rho\left(e^{-\mu_{j}(z)}\right)<m-1$ for $j=1,2, \ldots, n$, then by Lemma 4

$$
e^{-\mu_{0}(z)}=\cdots=e^{-\mu_{n}(z)} \equiv 0,
$$

which is a contradiction. We thus proved that $\operatorname{deg} \alpha=\operatorname{deg} \gamma=1$. Then $\operatorname{deg} \beta \leq 1$ for $\beta=$ $\alpha+\gamma$.

Without loss of generality, we may assume that

$$
\alpha(z)=\lambda_{1} z+\eta_{1}, \quad \beta(z)=\lambda_{2} z+\eta_{2},
$$

where $\lambda_{1}(\neq 0), \lambda_{2}, \eta_{1}, \eta_{2}$ are constants.

Thus $\alpha(z+j c)=\alpha(z)+j \lambda_{1} c$,

and

$$
\begin{aligned}
\Delta_{c}^{n} e^{-\alpha} & =\sum_{i=1}^{n} C_{n}^{i}(-1)^{n-i} e^{-\alpha_{i c}} \\
& =\sum_{i=1}^{n} C_{n}^{i}(-1)^{n-i} e^{-\alpha} e^{-i \lambda_{1} c} \\
& =e^{-\alpha} \sum_{i=1}^{n} C_{n}^{i}(-1)^{n-i} e^{-i \lambda_{1} c} \\
& =e^{-\alpha}\left(e^{-\lambda_{1} c}-1\right)^{n} .
\end{aligned}
$$

For $\Delta_{c}^{n} e^{-\alpha} \equiv 0$, that is, $e^{-\alpha}\left(e^{-\lambda_{1} c}-1\right)^{n} \equiv 0$, then $e^{\lambda_{1} c} \equiv 1$.

Therefore,

$$
e^{\alpha(z+i c)}=e^{\alpha(z)+i \lambda_{1} c}=e^{\alpha(z)}
$$

which implies that $e^{\alpha(z)}$ is a periodic function with period $c$.

We claim that $e^{\beta(z)}$ is also a periodic function with period $c$, and $e^{\lambda_{2} c}=1$.

Otherwise, suppose that $e^{\lambda_{2} c} \neq 1$.

Since $\gamma(z)=\beta(z)-\alpha(z)=\left(\lambda_{2}-\lambda_{1}\right) z+\left(\eta_{2}-\eta_{1}\right)$, and $\gamma_{j c}(z)=\gamma(z)+j c\left(\lambda_{2}-\lambda_{1}\right)$.

Then by equation (2.11), we have

$$
\alpha_{1} e^{\left(\lambda_{2}-\lambda_{1}\right) z}+\alpha_{2} e^{2\left(\lambda_{2}-\lambda_{1}\right) z}+\cdots+\alpha_{n} e^{n\left(\lambda_{2}-\lambda_{1}\right) z}=e^{\lambda_{1} z+\eta_{1}},
$$

where $\alpha_{1}, \alpha_{2}, \ldots, \alpha_{n}\left(=C_{n}^{n}=1\right)$ are constants.

Noticing that $e^{\lambda_{1} c}=1$, thus

$$
\begin{aligned}
\alpha_{1}= & {\left[C_{n}^{1}(-1)^{n-1}\right.} \\
& +C_{n}^{2}(-1)^{n-2}+C_{n}^{2}(-1)^{n-2} e^{\lambda_{2} c} \\
& +C_{n}^{3}(-1)^{n-3}+C_{n}^{3}(-1)^{n-3} e^{\lambda_{2} c}+C_{n}^{3}(-1)^{n-3} e^{2 \lambda_{2} c}+\cdots \\
& \left.+C_{n}^{n}(-1)^{n-n}+C_{n}^{n}(-1)^{n-n} e^{\lambda_{2} c}+\cdots+C_{n}^{n}(-1)^{n-n} e^{(n-1) \lambda_{2} c}\right] e^{\eta_{2}-\eta_{1}} \\
= & {\left[C_{n}^{1}(-1)^{n-1} \frac{e^{\lambda_{2} c}-1}{e^{\lambda_{2} c}-1}+C_{n}^{2}(-1)^{n-2} \frac{e^{2 \lambda_{2} c}-1}{e^{\lambda_{2} c}-1}+\cdots\right.}
\end{aligned}
$$




$$
\begin{aligned}
& \left.+C_{n}^{n}(-1)^{n-n} \frac{e^{n \lambda_{2} c-1}}{e^{\lambda_{2} c}-1}\right] e^{\eta_{2}-\eta_{1}} \\
= & {\left[\sum_{i=0}^{n} C_{n}^{i}(-1)^{n-i} e^{i \lambda_{2} c}-(-1)^{n}-\sum_{i=1}^{n} C_{n}^{i}(-1)^{n-i}\right] \frac{e^{\eta_{2}-\eta_{1}}}{e^{\lambda_{2} c}-1} } \\
= & {\left[\sum_{i=0}^{n} C_{n}^{i}(-1)^{n-i} e^{i \lambda_{2} c}-\sum_{i=0}^{n} C_{n}^{i}(-1)^{n-i}\right] \frac{e^{\eta_{2}-\eta_{1}}}{e^{\lambda_{2} c}-1} } \\
= & \left(e^{\lambda_{2} c}-1\right)^{n} \frac{e^{\eta_{2}-\eta_{1}}}{e^{\lambda_{2} c}-1} \\
= & \left(e^{\lambda_{2} c}-1\right)^{n-1} e^{\eta_{2}-\eta_{1}} .
\end{aligned}
$$

If $\lambda_{1}=\lambda_{2}$, then $e^{\beta(z+j c)}=e^{\beta(z)}$, a contradiction.

If $\lambda_{1} \neq \lambda_{2}$, we can rewrite equation (2.13) as follows:

$$
\alpha_{1} e^{\left(\lambda_{2}-2 \lambda_{1}\right) z}+\alpha_{2} e^{\left(2 \lambda_{2}-3 \lambda_{1}\right) z}+\cdots+\alpha_{n} e^{\left(n \lambda_{2}-(n+1) \lambda_{1}\right) z}=e^{\eta_{1}}
$$

If $i \lambda_{2}-(i+1) \lambda_{1} \neq 0$ for each $i=1,2, \ldots, n$, it is obvious that, for $1 \leq l<m \leq n$,

$$
\rho\left(e^{\left[m \lambda_{2}-(m+1) \lambda_{1}-l \lambda_{2}+(l+1) \lambda_{1}\right] z}\right)=\rho\left(e^{(m-l)\left(\lambda_{2}-\lambda_{1}\right) z}\right)=1
$$

then by Lemma 4, we get

$$
\alpha_{i}=e^{\eta_{1}}=0, \quad i=1,2, \ldots, n,
$$

which is a contradiction.

So there exists one integer $j \in\{1,2, \ldots, n\}$ such that $j \lambda_{2}-(j+1) \lambda_{1}=0$. Without loss of generality, we assume that $n \lambda_{2}-(n+1) \lambda_{1}=0$.

Thus (2.14) can be rewritten as follows:

$$
\alpha_{1} e^{\left(\lambda_{2}-2 \lambda_{1}\right) z}+\alpha_{2} e^{\left(2 \lambda_{2}-3 \lambda_{1}\right) z}+\cdots+\alpha_{n-1} e^{\left((n-1) \lambda_{2}-n \lambda_{1}\right) z}=e^{\eta_{1}}-\alpha_{n}
$$

Since $\alpha_{n}=1$, thus $e^{\eta_{1}}-\alpha_{n} \neq 0$. We also can deduce by Lemma 4 that $\alpha_{1}=0$, which means $\left(e^{\lambda_{2} c}-1\right)^{n-1} e^{\eta_{2}-\eta_{1}}=0$, thus $e^{\lambda_{2} c}=1$, a contradiction.

Thus

$$
e^{\beta(z+j c)}=e^{\beta(z)} e^{j \lambda_{2} c}=e^{\beta(z)},
$$

so $e^{\beta(z)}$ is also a periodic function with period $c$.

Therefore, we can deduce by equation (2.1) that

$$
\begin{aligned}
\Delta_{c}^{n+1} f & =\Delta_{c}^{n} f(z+c)-\Delta_{c}^{n} f(z) \\
& =e^{\alpha} \Delta_{c} f-\left(e^{\alpha}-1\right) \Delta_{c} P \\
& =e^{\beta} f-\left(e^{\beta}-1\right) P,
\end{aligned}
$$

thus

$$
\Delta_{c} f=e^{\beta-\alpha} f+\left(1-e^{-\alpha}\right) \Delta_{c} P-\left(e^{\beta-\alpha}-e^{-\alpha}\right) P .
$$


By mathematical induction, we have

$$
\begin{aligned}
\Delta_{c}^{2} f= & e^{2(\beta-\alpha)} f+\left(1-e^{-\alpha}\right) \Delta_{c}^{2} P+\left[e^{\beta-\alpha}\left(1-e^{-\alpha}\right)-\left(e^{\beta-\alpha}-e^{-\alpha}\right)\right] \\
& \times \Delta_{c} P-e^{\beta-\alpha}\left(e^{\beta-\alpha}-e^{-\alpha}\right) P, \\
\Delta_{c}^{3} f= & e^{3(\beta-\alpha)} f+\left(1-e^{-\alpha}\right) \Delta_{c}^{3} P+\left[e^{\beta-\alpha}\left(1-e^{-\alpha}\right)-\left(e^{\beta-\alpha}-e^{-\alpha}\right)\right] \\
& \times \Delta_{c}^{2} P+\left[e^{\beta-\alpha}\left(e^{\beta-\alpha}-e^{-\alpha}\right)-e^{2(\beta-\alpha)}\left(1-e^{-\alpha}\right)\right] \Delta_{c} P \\
& -e^{2(\beta-\alpha)}\left(e^{\beta-\alpha}-e^{-\alpha}\right) P, \\
\cdots, \quad & \\
\Delta_{c}^{n} f= & e^{n(\beta-\alpha)} f+\left(1-e^{-\alpha}\right) \Delta_{c}^{n} P+\left[e^{\beta-\alpha}\left(1-e^{-\alpha}\right)-\left(e^{\beta-\alpha}-e^{-\alpha}\right)\right] \\
& \times \Delta_{c}^{n-1} P+\cdots+\left[e^{(n-1)(\beta-\alpha)}\left(1-e^{-\alpha}\right)-e^{(n-2)(\beta-\alpha)}\left(e^{\beta-\alpha}-e^{-\alpha}\right)\right] \\
& \times \Delta_{c} P-e^{(n-1)(\beta-\alpha)}\left(e^{\beta-\alpha}-e^{-\alpha}\right) P \\
= & e^{n(\beta-\alpha)} f+\left(1-e^{-\alpha}\right) \sum_{i=0}^{n-1} e^{i(\beta-\alpha)} \Delta_{c}^{n-i} P \\
& -\left(e^{\beta-\alpha}-e^{-\alpha}\right) \sum_{i=0}^{n-1} e^{i(\beta-\alpha)} \Delta_{c}^{n-i-1} P .
\end{aligned}
$$

And by (2.1)

$$
\Delta_{c}^{n} f=e^{\alpha} f-\left(e^{\alpha}-1\right) P
$$

Therefore

$$
\begin{aligned}
e^{\alpha} f-\left(e^{\alpha}-1\right) P= & e^{n(\beta-\alpha)} f+\left(1-e^{-\alpha}\right) \sum_{i=0}^{n-1} e^{i(\beta-\alpha)} \Delta_{c}^{n-i} P \\
& -\left(e^{\beta-\alpha}-e^{-\alpha}\right) \sum_{i=0}^{n-1} e^{i(\beta-\alpha)} \Delta_{c}^{n-i-1} P .
\end{aligned}
$$

Hence

$$
\begin{aligned}
{\left[e^{\alpha}-e^{n(\beta-\alpha)}\right] f=} & \left(1-e^{-\alpha}\right) \sum_{i=0}^{n-1} e^{i(\beta-\alpha)} \Delta_{c}^{n-i} P \\
& -\left(e^{\beta-\alpha}-e^{-\alpha}\right) \sum_{i=0}^{n-1} e^{i(\beta-\alpha)} \Delta_{c}^{n-i-1} P+\left(e^{\alpha}-1\right) P .
\end{aligned}
$$

If $e^{\alpha}-e^{n(\beta-\alpha)} \not \equiv 0$, by the above equation, $T(r, f)=S(r, f)$, a contradiction.

Thus $e^{\alpha} \equiv e^{n(\beta-\alpha)}$, that is, $e^{n \beta} \equiv e^{(n+1) \alpha}$.

At the same time,

$$
\begin{aligned}
& \left(1-e^{-\alpha}\right) \sum_{i=0}^{n-1} e^{i(\beta-\alpha)} \Delta_{c}^{n-i} P-\left(1-e^{-\beta}\right) \sum_{i=0}^{n-1} e^{(i+1)(\beta-\alpha)} \Delta_{c}^{n-i-1} P \\
& \quad+\left(e^{\alpha}-1\right) P=0
\end{aligned}
$$




$$
\begin{aligned}
& \left(1-e^{-\alpha}\right) \Delta_{c}^{n} P+\left(1-e^{-\alpha}\right) \sum_{i=1}^{n-1} e^{i(\beta-\alpha)} \Delta_{c}^{n-i} P-\left(1-e^{-\beta}\right) \\
& \times \sum_{i=1}^{n-1} e^{i(\beta-\alpha)} \Delta_{c}^{n-i} P-e^{n(\beta-\alpha)}\left(1-e^{-\beta}\right) P+\left(e^{\alpha}-1\right) P=0 \\
& \left(1-e^{-\alpha}\right) \Delta_{c}^{n} P+\left(e^{-\beta}-e^{-\alpha}\right) \sum_{i=1}^{n-1} e^{i(\beta-\alpha)} \Delta_{c}^{n-i} P \\
& \quad+\left[\left(e^{\alpha}-1\right)-e^{n(\beta-\alpha)}\left(1-e^{-\beta}\right)\right] P=0 .
\end{aligned}
$$

If $P(z)$ is a constant, then $\Delta_{c}^{i} P=0$ for any $i \in \mathbb{N}^{+}$, then by (2.18),

$$
\left[\left(e^{\alpha}-1\right)-e^{n(\beta-\alpha)}\left(1-e^{-\beta}\right)\right] P=0
$$

thus

$$
e^{\alpha}-1=e^{n(\beta-\alpha)}\left(1-e^{-\beta}\right)
$$

Noticing that $e^{\alpha} \equiv e^{n(\beta-\alpha)}$, we have $e^{\alpha-\beta}=1$, which implies that $e^{\alpha}=e^{n(\beta-\alpha)}=1$, a contradiction.

So $P(z)$ is a nonconstant polynomial, we deduce by Nevanlinna's first fundamental theorem that

$$
\begin{aligned}
& T(r, P)=S\left(r, e^{\alpha}\right) ; \\
& T(r, P)=S\left(r, e^{\beta}\right) ; \\
& \operatorname{deg} \Delta_{c}^{i} P<\operatorname{deg} P
\end{aligned}
$$

for each $i \in \mathbb{N}^{+}$.

Since $\alpha(z)$ and $\beta(z)$ are both polynomials satisfying $e^{(n+1) \alpha}=e^{n \beta}$, set

$$
\alpha_{1}(z)=\frac{\alpha(z)}{n}, \quad \beta_{1}(z)=\frac{\beta(z)}{n+1},
$$

hence $\alpha_{1}(z), \beta_{1}(z)$ are polynomials of degree 1 satisfying

$$
e^{\alpha_{1}}=e^{\beta_{1}}, \quad e^{\alpha}=e^{n \alpha_{1}}, \quad e^{\beta}=e^{(n+1) \beta_{1}}
$$

and

$$
T(r, P)=S\left(r, e^{\alpha_{1}}\right), \quad T(r, P)=S\left(r, e^{\beta_{1}}\right) .
$$

Rewrite (2.18) as follows:

$$
\left(e^{n \alpha}-e^{(n-1) \alpha}\right) \Delta_{c}^{n} P+\left(e^{n \alpha-\beta}-e^{(n-1) \alpha}\right) \sum_{i=1}^{n-1} e^{i(\beta-\alpha)} \Delta_{c}^{n-i} P+\left(-e^{n \alpha}+e^{(n-1) \beta}\right) P=0
$$


thus,

$$
\begin{aligned}
e^{n \alpha}( & \left.\Delta_{c}^{n} P-P\right) \\
= & e^{(n-1) \alpha}\left(\Delta_{c}^{n} P-\Delta_{c}^{n-1} P\right)+e^{\beta+(n-2) \alpha}\left(\Delta_{c}^{n-1} P-\Delta_{c}^{n-2} P\right)+\cdots \\
& +e^{\alpha+(n-2) \beta}\left(\Delta_{c}^{2} P-\Delta_{c} P\right)+e^{(n-1) \beta}\left(P-\Delta_{c} P\right), \\
e^{n^{2} \alpha_{1}} & \left(\Delta_{c}^{n} P-P\right) \\
= & e^{\left(n^{2}-n\right) \alpha_{1}}\left(\Delta_{c}^{n} P-\Delta_{c}^{n-1} P\right)+e^{\left(n^{2}-n+1\right) \alpha_{1}}\left(\Delta_{c}^{n-1} P-\Delta_{c}^{n-2} P\right) \\
& +\cdots+e^{\left(n^{2}-2\right) \alpha_{1}}\left(\Delta_{c}^{2} P-\Delta_{c} P\right)+e^{\left(n^{2}-1\right) \alpha_{1}}\left(P-\Delta_{c} P\right) .
\end{aligned}
$$

Since $P(z) \not \equiv \Delta_{c}^{i} P$ for each $i \in \mathbb{N}^{+}$, by Nevanlinna's first fundamental theorem and (2.19),

$$
\begin{aligned}
n^{2} T & \left(r, e^{\alpha_{1}}\right)+S\left(r, e^{\alpha_{1}}\right) \\
= & T\left(r, e^{n^{2} \alpha_{1}}\left(\Delta_{c}^{n} P-P\right)\right) \\
= & T\left(r, e^{\left(n^{2}-n\right) \alpha_{1}}\left(\Delta_{c}^{n} P-\Delta_{c}^{n-1} P\right)+\cdots+e^{\left(n^{2}-2\right) \alpha_{1}}\left(\Delta_{c}^{2} P-\Delta_{c} P\right)\right. \\
& \left.+e^{\left(n^{2}-1\right) \alpha_{1}}\left(P-\Delta_{c} P\right)\right) \\
\leq & \left(n^{2}-1\right) T\left(r, e^{\alpha_{1}}\right)+S\left(r, e^{\alpha}\right),
\end{aligned}
$$

a contradiction.

Case 2.2. $\gamma(z)$ is a constant.

We claim that $\alpha(z)$ is also a constant.

Suppose that $\operatorname{deg} \alpha(z) \geq 1$, thus $\operatorname{deg}\left(\alpha-\alpha_{i c}\right) \leq \operatorname{deg} \alpha-1$. Notice that $\beta=\alpha+\gamma$ and $\gamma_{k c}=$ $\beta_{k c}-\alpha_{k c}=\gamma$. Then, by equation (2.10),

$\operatorname{deg} \alpha \leq \operatorname{deg} \alpha-1$,

which is impossible.

Hence $\alpha$ is a constant, so is $\beta$ for $\beta=\alpha+\gamma$.

Using the same method as the previous proof, we can deduce by equation (2.1) that

$$
\Delta_{c} f=e^{\beta-\alpha} f+\left(1-e^{-\alpha}\right) \Delta_{c} P-\left(e^{\beta-\alpha}-e^{-\alpha}\right) P .
$$

Thus

$$
\begin{aligned}
\Delta_{c}^{n} f= & e^{n(\beta-\alpha)} f+\left(1-e^{-\alpha}\right) \sum_{i=0}^{n-1} e^{i(\beta-\alpha)} \Delta_{c}^{n-i} P \\
& -\left(e^{\beta-\alpha}-e^{-\alpha}\right) \sum_{i=0}^{n-1} e^{i(\beta-\alpha)} \Delta_{c}^{n-i-1} P, \\
& =e^{\alpha} f-\left(e^{\alpha}-1\right) P(z), \\
\Delta_{c}^{n+1} f & =e^{(n+1)(\beta-\alpha)} f+\left(1-e^{-\alpha}\right) \sum_{i=0}^{n} e^{i(\beta-\alpha)} \Delta_{c}^{n+1-i} P
\end{aligned}
$$


Lin et al. Advances in Difference Equations

(2018) 2018:428

Page 15 of 27

$$
\begin{aligned}
& -\left(e^{\beta-\alpha}-e^{-\alpha}\right) \sum_{i=0}^{n} e^{i(\beta-\alpha)} \Delta_{c}^{n-i} P \\
= & e^{\beta} f-\left(e^{\beta}-1\right) P(z) .
\end{aligned}
$$

Then

$$
\begin{gathered}
\left(e^{\alpha}-e^{n(\beta-\alpha)}\right) f=\left(e^{\alpha}-1\right) P(z)+\left(1-e^{-\alpha}\right) \sum_{i=0}^{n-1} e^{i(\beta-\alpha)} \Delta_{c}^{n-i} P \\
-\left(e^{\beta-\alpha}-e^{-\alpha}\right) \sum_{i=0}^{n-1} e^{i(\beta-\alpha)} \Delta_{c}^{n-i-1} P, \\
\left(e^{\beta}-e^{(n+1)(\beta-\alpha)}\right) f= \\
-\left(e^{\beta}-1\right) P(z)+\left(1-e^{-\alpha}\right) \sum_{i=0}^{n} e^{i(\beta-\alpha)} \Delta_{c}^{n+1-i} P \\
-\left(e^{\beta-\alpha}-e^{-\alpha}\right) \sum_{i=0}^{n} e^{i(\beta-\alpha)} \Delta_{c}^{n-i} P .
\end{gathered}
$$

If either $e^{\alpha} \neq e^{n(\beta-\alpha)}$, or $e^{\beta} \neq e^{(n+1)(\beta-\alpha)}$, we deduce by $(2.22)$ that $T(r, f)=S(r, f)$, a contradition. Thus

$$
e^{\alpha}=e^{n(\beta-\alpha)}, \quad e^{\beta}=e^{(n+1)(\beta-\alpha)} .
$$

By (2.1),

$$
\frac{\Delta_{c}^{n+1} f-P}{\Delta_{c}^{n} f-P}=e^{\beta-\alpha}
$$

then

$$
\Delta_{c}^{n+1} f=e^{\beta-\alpha} \Delta_{c}^{n} f+\left(1-e^{\beta-\alpha}\right) P .
$$

We claim that $\Delta_{c}^{n+1} f \equiv \Delta_{c}^{n} f$.

Suppose that $\Delta_{c}^{n+1} f \not \equiv \Delta_{c}^{n} f$, then by (2.21) and (2.23), we have

$$
\begin{aligned}
& e^{(n+1)(\beta-\alpha)} f+\left(1-e^{-\alpha}\right) \sum_{i=0}^{n} e^{i(\beta-\alpha)} \Delta_{c}^{n+1-i} P-\left(e^{\beta-\alpha}-e^{-\alpha}\right) \sum_{i=0}^{n} e^{i(\beta-\alpha)} \Delta_{c}^{n-i} P \\
& =e^{\beta-\alpha}\left[e^{n(\beta-\alpha)} f+\left(1-e^{-\alpha}\right) \sum_{i=0}^{n-1} e^{i(\beta-\alpha)} \Delta_{c}^{n-i} P-\left(e^{\beta-\alpha}-e^{-\alpha}\right)\right. \\
& \left.\quad \times \sum_{i=0}^{n-1} e^{i(\beta-\alpha)} \Delta_{c}^{n-i-1} P\right]+\left(1-e^{\beta-\alpha}\right) P,
\end{aligned}
$$

and

$$
\left(1-e^{-\alpha}\right) \sum_{i=0}^{n} e^{i(\beta-\alpha)} \Delta_{c}^{n+1-i} P-\left(e^{\beta-\alpha}-e^{-\alpha}\right) \sum_{i=0}^{n} e^{i(\beta-\alpha)} \Delta_{c}^{n-i} P
$$




$$
\begin{aligned}
= & \left(1-e^{-\alpha}\right) \sum_{i=0}^{n-1} e^{(i+1)(\beta-\alpha)} \Delta_{c}^{n-i} P-\left(e^{\beta-\alpha}-e^{-\alpha}\right) \sum_{i=0}^{n-1} e^{(i+1)(\beta-\alpha)} \Delta_{c}^{n-i-1} P \\
& +\left(1-e^{\beta-\alpha}\right) P .
\end{aligned}
$$

Hence,

$$
\left(1-e^{-\alpha}\right) \Delta_{c}^{n+1} P-\left(e^{\beta-\alpha}-e^{-\alpha}\right) \Delta_{c}^{n} P=\left(1-e^{\beta-\alpha}\right) P .
$$

We claim that $1-e^{\beta-\alpha}=0$.

Suppose $1-e^{\beta-\alpha} \neq 0$.

If $P(z)$ is a constant, by (2.24), we have $P \equiv 0$, a contradiction.

If $P(z)$ is a nonconstant polynomial, the degree of the left-hand side of (2.24) is less than that of the right-hand side, a contradiction.

Thus $e^{\beta-\alpha}=1$, then $e^{\alpha}=e^{n(\beta-\alpha)}=1, e^{\beta}=e^{(n+1)(\beta-\alpha)}=1$.

Then, by (2.23), we have proved $\Delta_{c}^{n} f \equiv \Delta_{c}^{n+1} f$, which is a contradiction to our assumption.

Thus $\Delta_{c}^{n} f \equiv \Delta_{c}^{n+1} f$, and $e^{\alpha}=e^{n(\beta-\alpha)}=1, e^{\beta}=e^{(n+1)(\beta-\alpha)}=1$.

By (2.20), we have

$$
f \equiv \Delta_{c} f
$$

hence Lemma 7 is proved.

\section{Proof of Theorem 8}

Proof Since $f, \Delta_{c}^{n} f$, and $\Delta_{c}^{n+1} f$ share $\infty$ and $P(z) \mathrm{CM}$, and the order of $f$ is finite, then there exist two polynomials $\alpha(z)$ and $\beta(z)$ such that

$$
\left\{\begin{array}{l}
\frac{\Delta_{c}^{n} f(z)-P(z)}{f(z)-P(z)}=e^{\alpha(z),} \\
\frac{\Delta_{c}^{n+1} f(z)-P(z)}{f(z)-P(z)}=e^{\beta(z)} .
\end{array}\right.
$$

By Lemma 7, two cases will be considered in the following.

Case 1. $f$ is a rational function.

Using the same method as Lemma 7, we have

$$
f(z)=A P(z)
$$

where $A \neq 0,1$ is a constant, and $P(z)$ is a polynomial with $\operatorname{deg} P(z) \leq n-1$.

Case 2. $f$ is a transcendental meromorphic function, then $T(r, P)=S(r, f)$.

Set

$$
F(z)=f(z)-P(z)
$$

then $T(r, F)=T(r, f)+S(r, f)$, and $T(r, P)=S(r, F)$. 
It follows that

$$
\Delta_{c}^{n} f=\Delta_{c}^{n} F+\Delta_{c}^{n} P, \quad \Delta_{c}^{n+1} f=\Delta_{c}^{n+1} F+\Delta_{c}^{n+1} P .
$$

Then (3.1) can be written as

$$
\left\{\begin{array}{l}
\frac{\Delta_{c}^{n} F(z)+\Delta_{c}^{n} P(z)-P(z)}{F(z)}=e^{\alpha(z),} \\
\frac{\Delta_{c}^{n+1} F(z)+\Delta_{c}^{n+1} P(z)-P(z)}{F(z)}=e^{\beta(z)} .
\end{array}\right.
$$

Since $P(z)$ is a polynomial, then $\Delta_{c}^{n} P(z)-P(z) \not \equiv 0$ and $\Delta_{c}^{n+1} P(z)-P(z) \not \equiv 0$. Set

$$
\phi(z)=\frac{\left(P(z)-\Delta_{c}^{n+1} P(z)\right) \Delta_{c}^{n} F(z)-\left(P(z)-\Delta_{c}^{n} P(z)\right) \Delta_{c}^{n+1} F(z)}{F(z)} .
$$

Two cases will be discussed in the following.

Case 2.1. $\phi(z) \not \equiv 0$.

For $T(r, P)=S(r, F)$, by Lemma 2 , we have $m(r, \phi)=S(r, F)$.

Since

$$
\begin{aligned}
(P(z) & \left.-\Delta_{c}^{n+1} P(z)\right) \Delta_{c}^{n} F(z)-\left(P(z)-\Delta_{c}^{n} P(z)\right) \Delta_{c}^{n+1} F(z) \\
= & \left(P(z)-\Delta_{c}^{n+1} P(z)\right)\left(\Delta_{c}^{n} F(z)+\Delta_{c}^{n} P(z)-P(z)\right)-\left(P(z)-\Delta_{c}^{n} P(z)\right) \\
& \times\left(\Delta_{c}^{n+1} F(z)+\Delta_{c}^{n+1} P(z)-P(z)\right)-\left(P(z)-\Delta_{c}^{n+1} P(z)\right) \\
& \times\left(\Delta_{c}^{n} P(z)-P(z)\right)+\left(P(z)-\Delta_{c}^{n} P(z)\right)\left(\Delta_{c}^{n+1} P(z)-P(z)\right) \\
= & e^{\alpha(z)} F(z)\left(P(z)-\Delta_{c}^{n+1} P(z)\right)-e^{\beta(z)} F(z)\left(P(z)-\Delta_{c}^{n} P(z)\right),
\end{aligned}
$$

then we can rewrite $\phi(z)$ as follows:

$$
\phi(z)=\left(P(z)-\Delta_{c}^{n+1} P(z)\right) e^{\alpha(z)}-\left(P(z)-\Delta_{c}^{n} P(z)\right) e^{\beta(z)},
$$

and it is obvious that $N(r, \phi)=S(r, F)$. Hence $T(r, \phi)=S(r, F)$.

By (3.4),

$$
\left(P(z)-\Delta_{c}^{n+1} P(z)\right) \frac{e^{\alpha(z)}}{\phi(z)}=1+\left(P(z)-\Delta_{c}^{n} P(z)\right) \frac{e^{\beta(z)}}{\phi(z)},
$$

thus by Nevanlinna's second fundamental theorem,

$$
\begin{aligned}
T( & \left.r,\left(P(z)-\Delta_{c}^{n+1} P(z)\right) \frac{e^{\alpha(z)}}{\phi(z)}\right) \\
\leq & \bar{N}\left(r,\left(P(z)-\Delta_{c}^{n+1} P(z)\right) \frac{e^{\alpha(z)}}{\phi(z)}\right)+\bar{N}\left(r, \frac{\phi(z)}{\left(P(z)-\Delta_{c}^{n+1} P(z)\right) e^{\alpha(z)}}\right) \\
& +\bar{N}\left(r, \frac{1}{\left(P(z)-\Delta_{c}^{n+1} P(z)\right) \frac{e^{\alpha(z)}}{\phi(z)}-1}\right)+S\left(r,\left(P(z)-\Delta_{c}^{n+1} P(z)\right) \frac{e^{\alpha(z)}}{\phi(z)}\right) \\
\leq & S(r, F)+S\left(r,\left(P(z)-\Delta_{c}^{n+1} P(z)\right) \frac{e^{\alpha(z)}}{\phi(z)}\right) .
\end{aligned}
$$


Noticing that $T(r, \phi)=S(r, F)$ and $T(r, P)=S(r, F)$, we have $T\left(r, e^{\alpha(z)}\right)=S(r, F)$, so $T\left(r, e^{\alpha(z)}\right)=S(r, f)$.

And by (3.5), we also get $T\left(r, e^{\beta(z)}\right)=S(r, f)$.

Therefore, by Lemma 7, we have $f \equiv \Delta_{f} f$ since $f$ is a transcendental meromorphic function.

Case 2.2. $\phi(z) \equiv 0$.

By the definition of $\phi(z)$, we have

$$
\left(P(z)-\Delta_{c}^{n+1} P(z)\right) \Delta_{c}^{n} F(z)=\left(P(z)-\Delta_{c}^{n} P(z)\right) \Delta_{c}^{n+1} F(z) .
$$

For $F(z)=f(z)-P(z)$, thus

$$
\begin{aligned}
(P(z) & \left.-\Delta_{c}^{n+1} P(z)\right)\left(\Delta_{c}^{n} f(z)-\Delta_{c}^{n} P(z)\right) \\
= & \left(P(z)-\Delta_{c}^{n} P(z)\right)\left(\Delta_{c}^{n+1} f(z)-\Delta_{c}^{n+1} P(z)\right), \\
& \times\left(P(z)-\Delta_{c}^{n+1} P(z)\right) \Delta_{c}^{n} f-P \Delta_{c}^{n} P+P^{2} \\
= & \left(P(z)-\Delta_{c}^{n} P(z)\right) \Delta_{c}^{n+1} f(z)-P \Delta_{c}^{n+1} P+P^{2}, \\
& \times\left(P(z)-\Delta_{c}^{n+1} P(z)\right)\left(\Delta_{c}^{n} f-P\right)=\left(P(z)-\Delta_{c}^{n} P(z)\right)\left(\Delta_{c}^{n+1} f-P\right), \\
& \times\left(P(z)-\Delta_{c}^{n+1} P(z)\right) e^{\alpha}=\left(P(z)-\Delta_{c}^{n} P(z)\right) e^{\beta},
\end{aligned}
$$

which implies

$$
e^{\alpha-\beta}=\frac{P(z)-\Delta_{c}^{n} P(z)}{P(z)-\Delta_{c}^{n+1} P(z)} .
$$

Since $P(z)$ is a polynomial, then $e^{\alpha-\beta}$ is a constant. Assume that $e^{\alpha-\beta}=A$, then

$$
P(z)-\Delta_{c}^{n} P(z)=A\left(P(z)-\Delta_{c}^{n+1} P(z)\right) .
$$

Comparing the coefficients of both sides of equation (3.8), $A=1$, then $\Delta_{c}^{n} P \equiv \Delta_{c}^{n+1} P$. Since $P(z)$ is a polynomial, thus $\Delta_{c}^{n} P \equiv \Delta_{c}^{n+1} P \equiv 0$, and $P(z)$ is a polynomial of degree less than $n$.

By (3.6), we have $\Delta_{c}^{n} F(z)=\Delta_{c}^{n+1} F(z)$. Thus by (3.1), $e^{\alpha(z)}=e^{\beta(z)}$, then (3.2) can be rewritten as follows:

$$
\left\{\begin{array}{l}
\frac{\Delta_{c}^{n} F-P}{F}=e^{\alpha(z),} \\
\frac{\Delta_{c}^{n+1} F-P}{F}=e^{\alpha(z)} .
\end{array}\right.
$$

Hence

$$
\left\{\begin{array}{l}
\Delta_{c}^{n} F=e^{\alpha(z)} F+P, \\
\Delta_{c}^{n+1} F=e^{\alpha(z)} F+P .
\end{array}\right.
$$

By the definition of $n$th difference,

$$
\Delta_{c}^{n+1} F=\Delta_{c}^{n} F(z+c)-\Delta_{c}^{n} F(z)
$$




$$
\begin{aligned}
& =e^{\alpha_{c}} F_{c}+P_{c}-e^{\alpha} F-P \\
& =e^{\alpha(z)} F+P .
\end{aligned}
$$

Thus

$$
F_{c}=2 e^{\alpha-\alpha_{c}} F+\left(2 P-P_{c}\right) e^{-\alpha_{c}} .
$$

Then we can deduce that

$$
\begin{aligned}
& F_{2 c}=2^{2} e^{\alpha-\alpha_{2 c}} F+\left(2^{2} P-P_{2 c}\right) e^{-\alpha_{2 c}}, \\
& F_{3 c}=2^{3} e^{\alpha-\alpha_{3 c}} F+\left(2^{3} P-P_{3 c}\right) e^{-\alpha_{3 c}}, \\
& \cdots
\end{aligned}
$$

By mathematical induction,

$$
F_{k c}=2^{k} e^{\alpha-\alpha_{k c}} F+\left(2^{k} P-P_{k c}\right) e^{-\alpha_{k c}} .
$$

Thus

$$
\begin{aligned}
\Delta_{c}^{n} F & =\sum_{i=0}^{n} C_{n}^{i}(-1)^{n-i} F_{i c} \\
& =\sum_{i=0}^{n} C_{n}^{i}(-1)^{n-i}\left(2^{i} e^{\alpha-\alpha_{i c}} F+\left(2^{i} P-P_{i c}\right) e^{-\alpha_{i c}}\right) \\
& =e^{\alpha} F+P
\end{aligned}
$$

then

$$
\begin{aligned}
& {\left[\sum_{i=0}^{n} C_{n}^{i}(-1)^{n-i} 2^{i} e^{\alpha-\alpha_{i c}}-e^{\alpha}\right] F} \\
& \quad+\left[\sum_{i=0}^{n} C_{n}^{i}(-1)^{n-i}\left(2^{i} P-P_{i c}\right) e^{-\alpha_{i c}}-P\right]=0 .
\end{aligned}
$$

Using the same method, we have

$$
\begin{aligned}
& {\left[\sum_{i=0}^{n+1} C_{n+1}^{i}(-1)^{n+1-i} 2^{i} e^{\alpha-\alpha_{i c}}-e^{\alpha}\right] F} \\
& \quad+\left[\sum_{i=0}^{n+1} C_{n+1}^{i}(-1)^{n+1-i}\left(2^{i} P-P_{i c}\right) e^{-\alpha_{i c}}-P\right]=0 .
\end{aligned}
$$

Define that

$$
A_{n}=\sum_{i=0}^{n} C_{n}^{i}(-1)^{n-i} 2^{i} e^{\alpha-\alpha_{i c}}-e^{\alpha},
$$




$$
\begin{aligned}
& B_{n}=\sum_{i=0}^{n} C_{n}^{i}(-1)^{n-i}\left(2^{i} P-P_{i c}\right) e^{-\alpha_{i c}}-P, \\
& A_{n+1}=\sum_{i=0}^{n+1} C_{n+1}^{i}(-1)^{n+1-i} 2^{i} e^{\alpha-\alpha_{i c}}-e^{\alpha}, \\
& B_{n+1}=\sum_{i=0}^{n+1} C_{n+1}^{i}(-1)^{n+1-i}\left(2^{i} P-P_{i c}\right) e^{-\alpha_{i c}}-P .
\end{aligned}
$$

Let $v=P e^{-\alpha}$, and note that

$$
\begin{aligned}
e^{\alpha} B_{n} & =\left[\sum_{i=0}^{n} C_{n}^{i}(-1)^{n-i} 2^{i} e^{\alpha-\alpha_{i c}}-e^{\alpha}\right] P-e^{\alpha} \sum_{i=0}^{n} C_{n}^{i}(-1)^{n-i} P_{i c} e^{-\alpha_{i c}} \\
& =A_{n} P-e^{\alpha} \Delta_{c}^{n} \nu,
\end{aligned}
$$

then

$$
B_{n}=e^{-\alpha} A_{n} P-\Delta_{c}^{n} \nu .
$$

Thus

$$
B_{n+1}=e^{-\alpha} A_{n+1} P-\Delta_{c}^{n+1} v .
$$

By (3.13) and (3.14), we have

$$
A_{n} B_{n+1}-A_{n+1} B_{n} \equiv 0,
$$

that is,

$$
\begin{aligned}
& A_{n}\left(e^{-\alpha} A_{n+1} P-\Delta_{c}^{n+1} v\right)-A_{n+1}\left(e^{-\alpha} A_{n} P-\Delta_{c}^{n} v\right) \\
& \quad=A_{n+1} \Delta_{c}^{n} v-A_{n} \Delta_{c}^{n+1} v=0 .
\end{aligned}
$$

Then

$$
\begin{aligned}
& {\left[\sum_{i=0}^{n+1} C_{n+1}^{i}(-1)^{n+1-i} 2^{i} e^{\alpha-\alpha_{i c}}-e^{\alpha}\right] \sum_{i=0}^{n} C_{n}^{i}(-1)^{n-i} P_{i c} e^{-\alpha_{i c}}} \\
& -\left[\sum_{i=0}^{n} C_{n}^{i}(-1)^{n-i} 2^{i} e^{\alpha-\alpha_{i c}}-e^{\alpha}\right] \sum_{i=0}^{n+1} C_{n+1}^{i}(-1)^{n+1-i} P_{i c} e^{-\alpha_{i c}}=0, \\
& \Delta_{c}^{n} \nu \sum_{i=0}^{n+1} C_{n+1}^{i}(-1)^{n+1-i} 2^{i} e^{-\alpha_{i c}}-\Delta_{c}^{n+1} \nu \sum_{i=0}^{n} C_{n}^{i}(-1)^{n-i} 2^{i} e^{-\alpha_{i c}} \\
& \quad=\Delta_{c}^{n} \nu-\Delta_{c}^{n+1} v \\
& 2^{n+1} \Delta_{c}^{n} \nu e^{-\alpha_{(n+1) c}}+\sum_{i=0}^{n}\left(\Delta_{c}^{n} \nu C_{n+1}^{i}+\Delta_{c}^{n+1} \nu C_{n}^{i}\right)(-1)^{n+1-i} 2^{i} e^{-\alpha_{i c}} \\
& \quad=\Delta_{c}^{n} \nu-\Delta_{c}^{n+1} \nu .
\end{aligned}
$$


Hence,

$$
\begin{aligned}
& 2^{n+1} \Delta_{c}^{n} \nu+\sum_{i=0}^{n}\left(\Delta_{c}^{n} \nu C_{n+1}^{i}+\Delta_{c}^{n+1} v C_{n}^{i}\right)(-1)^{n+1-i} 2^{i} e^{\alpha_{(n+1) c}-\alpha_{i c}} \\
& \quad=e^{\alpha(n+1) c}\left(\Delta_{c}^{n} v-\Delta_{c}^{n+1} v\right)
\end{aligned}
$$

Set

$$
\gamma_{i}(z)=(-1)^{n+1-i} 2^{i} e^{\alpha_{(n+1) c}-\alpha_{i c}}, \quad i=1,2, \ldots, n,
$$

and

$$
\begin{aligned}
& \gamma_{n+1}(z) \\
& =\left(\Delta_{c}^{n} v-\Delta_{c}^{n+1} v\right) e^{\alpha_{(n+1) c}} \\
& =\sum_{i=0}^{n} C_{n}^{i}(-1)^{n-i} P_{i c} e^{\alpha_{(n+1) c}-\alpha_{i c}}-\sum_{i=0}^{n+1} C_{n+1}^{i}(-1)^{n+1-i} P_{i c} e^{\alpha_{(n+1) c}-\alpha_{i c}} .
\end{aligned}
$$

It is obvious that $\rho\left(\gamma_{i}(z)\right) \leq \operatorname{deg} \alpha-1$ for each $i \in\{1,2, \ldots, n, n+1\}$. Let

$$
M(z)=\sum_{i=0}^{n} C_{n+1}^{i} \gamma_{i}(z)+2^{n+1}, \quad N(z)=\sum_{i=0}^{n} C_{n}^{i} \gamma_{i}(z)
$$

then $\rho(M(z)) \leq \operatorname{deg} \alpha-1$, and $\rho(N(z)) \leq \operatorname{deg} \alpha-1$.

Thus by (3.15)

$$
M(z) \sum_{i=0}^{n} C_{n}^{i}(-1)^{n-i} P_{i c} e^{-\alpha_{i c}}+N(z) \sum_{i=0}^{n+1} C_{n+1}^{i}(-1)^{n+1-i} P_{i c} e^{-\alpha_{i c}}=\gamma_{n+1}(z)
$$

Then we rewrite (3.16) as follows:

$$
d_{n+1}(z) e^{-\alpha(z+(n+1) c)}+d_{n}(z) e^{-\alpha(z+n c)}+\cdots+d_{0}(z) e^{-\alpha(z)}=\gamma_{n+1}(z)
$$

where $d_{n+1}(z)=N(z) P_{(n+1) c}, d_{n}(z)=(M(z)-(n+1) N(z)) P_{n c}, \ldots, d_{0}(z)=\left((-1)^{n+1} N(z)+\right.$ $\left.(-1)^{n} M(z)\right) P$.

Next, we consider three cases.

Case 2.2.1. $\operatorname{deg} \alpha \geq 2$.

By the definition of $M(z), N(z)$, it can be proved that $\rho\left(d_{i}(z)\right) \leq \operatorname{deg} \alpha-1, i=0,1, \ldots, n$, $n+1$.

Since

$$
\begin{aligned}
\gamma_{n+1} & (z) \\
& =\left(\Delta_{c}^{n} \nu-\Delta_{c}^{n+1} \nu\right) e^{\alpha_{(n+1) c}} \\
& =\sum_{i=0}^{n} C_{n}^{i}(-1)^{n-i} P_{i c} e^{\alpha_{(n+1) c}-\alpha_{i c}}-\sum_{i=0}^{n+1} C_{n+1}^{i}(-1)^{n+1-i} P_{i c} e^{\alpha_{(n+1) c}-\alpha_{i c}}
\end{aligned}
$$




$$
\begin{aligned}
= & 2(-1)^{n} P e^{\alpha_{(n+1) c}-\alpha}+\left(C_{n+1}^{1}+C_{n}^{1}\right)(-1)^{n-1} P_{c} e^{\alpha_{(n+1) c}-\alpha_{c}}+\left(C_{n+1}^{2}+C_{n}^{2}\right)(-1)^{n-2} \\
& \times P_{2 c} e^{\alpha_{(n+1) c}-\alpha_{2 c}}+\cdots+\left(C_{n+1}^{n}+C_{n}^{n}\right) P_{n c} e^{\alpha_{(n+1) c}-\alpha_{n c}}-P_{(n+1) c}
\end{aligned}
$$

we claim that $\gamma_{n+1}(z) \not \equiv 0$. Otherwise,

$$
\begin{aligned}
P_{(n+1) c}= & 2(-1)^{n} P e^{\alpha_{(n+1) c}-\alpha}+\left(C_{n+1}^{1}+C_{n}^{1}\right)(-1)^{n-1} P_{c} e^{\alpha_{(n+1) c}-\alpha_{c}} \\
& +\left(C_{n+1}^{2}+C_{n}^{2}\right)(-1)^{n-2} P_{2 c} e^{\alpha_{(n+1) c}-\alpha_{2 c}}+\cdots \\
& +\left(C_{n+1}^{n}+C_{n}^{n}\right) P_{n c} e^{\alpha_{(n+1) c}-\alpha_{n c}} .
\end{aligned}
$$

Thus

$$
\begin{gathered}
2(-1)^{n} \frac{P}{P_{(n+1) c}} e^{\alpha_{(n+1) c}-\alpha}+(-1)^{n-1}\left(C_{n+1}^{1}+C_{n}^{1}\right) \frac{P_{c}}{P_{(n+1) c}} e^{\alpha_{(n+1) c}-\alpha_{c}} \\
+\cdots+\left(C_{n+1}^{n}+C_{n}^{n}\right) \frac{P_{n c}}{P_{(n+1) c}} e^{\alpha_{(n+1) c}-\alpha_{n c}}=1 .
\end{gathered}
$$

It is obvious that $(-1)^{n-i}\left(C_{n+1}^{i}+C_{n}^{i}\right) \frac{P_{i c}}{P_{(n+1) c}} e^{\alpha_{(n+1) c}-\alpha_{i c}}$ cannot be constants $C_{i}(\neq 0)$, and note that $\rho\left(e^{\alpha(n+1) c-\alpha_{i c}}\right) \geq 1$, hence by Lemma 3 ,

$$
\left(C_{n+1}^{n}+C_{n}^{n}\right) \frac{P_{n c}}{P_{(n+1) c}} e^{\alpha(n+1) c-\alpha_{n c}} \equiv 1
$$

which implies that $e^{\alpha_{(n+1) c}-\alpha_{n c}}=\frac{P_{(n+1) c}}{\left(C_{n+1}^{n}+C_{n}^{n}\right) P_{n c}}$, but it contradicts with the fact that $e^{\alpha_{(n+1) c}-\alpha_{n c}}$ is a transcendental entire function. Thus $\gamma_{n+1}(z) \not \equiv 0$.

Therefore, we rewrite (3.17) as follows:

$$
\frac{d_{n+1}(z)}{\gamma_{n+1}(z)} e^{-\alpha(z+(n+1) c)}+\frac{d_{n}(z)}{\gamma_{n+1}(z)} e^{-\alpha(z+n c)}+\cdots+\frac{d_{1}(z)}{\gamma_{n+1}(z)} e^{-\alpha(z+c)}+\frac{d_{0}(z)}{\gamma_{n+1}(z)} e^{-\alpha(z)}=1
$$

Obviously, $\frac{d_{j}(z) e^{-\alpha i c}}{\gamma_{n+1}(z)}$ cannot be constant $C_{j}(\neq 0)$, thus by Lemma 3, we have

$$
\frac{d_{0}(z) e^{-\alpha(z)}}{\gamma_{n+1}(z)} \equiv 0
$$

which means $e^{-\alpha(z)} \equiv \frac{\gamma_{n+1}(z)}{d_{0}(z)}$, a contradiction.

Case 2.2.2. $\operatorname{deg} \alpha=1$.

If $\gamma_{n+1}(z) \not \equiv 0$, by (3.17), by Lemma 3, and by the same method as Case 2.2.1, we also deduce a contradiction.

If $\gamma_{n+1}(z) \equiv 0$, without loss of generality, we assume that $\alpha(z)=\lambda z+\mu, \lambda \neq 0$, then

$$
\alpha_{(n+1) c}-\alpha_{i c}=(n+1-i) \lambda c .
$$

Thus

$$
\begin{aligned}
& \gamma_{n+1}(z) \\
& \quad=2(-1)^{n} P e^{(n+1) \lambda c}+\left(C_{n+1}^{1}+C_{n}^{1}\right)(-1)^{n-1} P_{c} e^{n \lambda c}+\left(C_{n+1}^{2}+C_{n}^{2}\right)
\end{aligned}
$$




$$
\begin{aligned}
& \times(-1)^{n-2} P_{2 c} e^{(n-1) \lambda c}+\cdots+\left(C_{n+1}^{n}+C_{n}^{n}\right) P_{n c} e^{\lambda c}-P_{(n+1) c} \\
= & \sum_{i=0}^{n}(-1)^{n-i}\left(C_{n}^{i}+C_{n+1}^{i}\right) P_{i c} e^{(n+1-i) \lambda c}-P_{(n+1) c} .
\end{aligned}
$$

Since $\operatorname{deg} P \leq n-1$, without loss of generality, we assume that

$$
P(z)=s z^{n-t}+P^{*}(z)
$$

where $t$ is a positive integer satisfying $1 \leq t<n$, and $s(\neq 0) \in \mathbb{C}$.

We assume that the coefficient of $z^{n-t}$ in $\gamma_{n+1}(z)$ is $A$, then

$$
A=s\left[\sum_{i=0}^{n}(-1)^{n-i}\left(C_{n+1}^{i}+C_{n}^{i}\right) e^{(n+1-i) \lambda c}-1\right]
$$

hence

$$
\begin{aligned}
A / s & =\sum_{i=0}^{n}(-1)^{n-i}\left(C_{n+1}^{i}+C_{n}^{i}\right) e^{(n+1-i) \lambda c}-1 \\
& =\sum_{i=0}^{n}(-1)^{n-i} C_{n+1}^{i} e^{(n+1-i) \lambda c}-1+\sum_{i=0}^{n}(-1)^{n-i} C_{n}^{i} e^{(n+1-i) \lambda c} \\
& =-\left(1-e^{\lambda c}\right)^{n+1}+e^{\lambda c} \sum_{i=0}^{n}(-1)^{n-i} C_{n}^{i} e^{(n-i) \lambda c} \\
& =\left(1-e^{\lambda c}\right)^{n+1}+e^{\lambda c}\left(1-e^{\lambda c}\right)^{n} \\
& =\left(1-e^{\lambda c}\right)^{n}\left(2 e^{\lambda c}-1\right) .
\end{aligned}
$$

Since $\gamma_{n+1}(z) \equiv 0$, then $A=0$, two subcases will be considered in the following.

Case 2.2.2.1. $1-e^{\lambda c}=0$, then $e^{\lambda c}=1$.

Hence

$$
e^{\alpha(z+i c)}=e^{\alpha(z)+i \lambda c}=e^{\alpha(z)},
$$

which means $e^{\alpha(z)}$ is a periodic function of period $c$, and $e^{\alpha_{i c}}=e^{\alpha}$.

Now we have

$$
\begin{aligned}
A_{n} & =\sum_{i=0}^{n} C_{n}^{i}(-1)^{n-i} 2^{i}-e^{\alpha}=1-e^{\alpha}, \\
B_{n} & =\sum_{i=0}^{n} C_{n}^{i}(-1)^{n-i}\left(2^{i} P-P_{i c}\right) e^{-\alpha}-P \\
& =e^{-\alpha}\left[P \sum_{i=0}^{n} C_{n}^{i}(-1)^{n-i} 2^{i}-\sum_{i=0}^{n} C_{n}^{i}(-1)^{n-i} P_{i c}\right]-P \\
& =e^{-\alpha} P-P=\left(e^{-\alpha}-1\right) P .
\end{aligned}
$$


By (3.13),

$$
\begin{aligned}
0 & =\left(1-e^{\alpha}\right) F+\left(e^{-\alpha}-1\right) P, \\
F & =\frac{1-e^{-\alpha}}{1-e^{\alpha}} P \\
& =-e^{-\alpha} P,
\end{aligned}
$$

then

$$
f=F+P=\left(1-e^{-\alpha}\right) P .
$$

Thus,

$$
\begin{aligned}
& \Delta_{c}^{n} F=e^{-\alpha} \Delta_{c}^{n} P \equiv 0, \\
& \Delta_{c}^{n+1} F=e^{-\alpha} \Delta_{c}^{n+1} P \equiv 0 .
\end{aligned}
$$

Therefore,

$$
\Delta_{c}^{n} f \equiv 0, \quad \Delta_{c}^{n+1} f \equiv 0 .
$$

It is obvious that $f, \Delta_{c}^{n} f, \Delta_{c}^{n+1} f$ share $\infty$ and $P(z) \mathrm{CM}$, thus

$$
f=\left(1-e^{-\alpha(z)}\right) P(z)
$$

where $e^{\alpha(z)}$ is a periodic function of period $c$ with $\operatorname{deg} \alpha=1$, and $P(z)$ is a polynomial with $\operatorname{deg} P \leq 1$.

Case 2.2.2.2. $1-2 e^{\lambda c}=0$, then $e^{\lambda c}=\frac{1}{2}$, and

$$
e^{\alpha_{i c}}=e^{\alpha} e^{i \lambda c}=2^{-i} e^{\alpha}
$$

Hence

$$
\begin{aligned}
A_{n} & =\sum_{i=0}^{n} C_{n}^{i}(-1)^{n-i} 2^{i} e^{\alpha} e^{-\alpha_{i c}}-e^{\alpha} \\
& =\sum_{i=0}^{n} C_{n}^{i}(-1)^{n-i} 4^{i}-e^{\alpha} \\
& =3^{n}-e^{\alpha}, \\
B_{n} & =\sum_{i=0}^{n} C_{n}^{i}(-1)^{n-i}\left(2^{i} P-P_{i c}\right) e^{-\alpha_{i c}}-P \\
& =e^{-\alpha}\left[P \sum_{i=0}^{n} C_{n}^{i}(-1)^{n-i} 4^{i}-\sum_{i=0}^{n} C_{n}^{i}(-1)^{n-i} P_{i c} 2^{i}\right]-P \\
& =3^{n} P e^{-\alpha}-e^{-\alpha} \sum_{i=0}^{n} C_{n}^{i}(-1)^{n-i} P_{i c} 2^{i}-P,
\end{aligned}
$$




$$
\begin{aligned}
A_{n+1} & =\sum_{i=0}^{n+1} C_{n+1}^{i}(-1)^{n+1-i} 2^{i} e^{\alpha} e^{-\alpha_{i c}}-e^{\alpha} \\
& =\sum_{i=0}^{n+} C_{n+1}^{i}(-1)^{n-i} 4^{i}-e^{\alpha} \\
& =3^{n+1}-e^{\alpha}, \\
B_{n+1} & =\sum_{i=0}^{n+1} C_{n+1}^{i}(-1)^{n+1-i}\left(2^{i} P-P_{i c}\right) e^{-\alpha_{i c}}-P \\
& =\sum_{i=0}^{n+1} C_{n+1}^{i}(-1)^{n+1-i} 4^{i}\left(e^{-\alpha} P-e^{-\alpha} P_{i c} 2^{i}\right)-P \\
& =e^{-\alpha} P \sum_{i=0}^{n+1} C_{n+1}^{i}(-1)^{n+1-i} 4^{i}-e^{-\alpha} \sum_{i=0}^{n+1} C_{n+1}^{i}(-1)^{n+1-i} P_{i c} 2^{i}-P \\
& =3^{n+1} P e^{-\alpha}-e^{-\alpha} \sum_{i=0}^{n+1} C_{n+1}^{i}(-1)^{n+1-i} P_{i c} 2^{i}-P .
\end{aligned}
$$

By (3.13) and (3.14), we have

$$
\begin{aligned}
& A_{n} B_{n+1}=B_{n} A_{n+1}, \\
& \left(3^{n}-e^{\alpha}\right)\left(3^{n+1} P e^{-\alpha}-e^{-\alpha} \sum_{i=0}^{n+1} C_{n+1}^{i}(-1)^{n+1-i} P_{i c} 2^{i}-P\right) \\
& =\left(3^{n+1}-e^{\alpha}\right)\left(3^{n} P e^{-\alpha}-e^{-\alpha} \sum_{i=0}^{n} C_{n}^{i}(-1)^{n-i} P_{i c} 2^{i}-P\right), \\
& \sum_{i=0}^{n+1} C_{n+1}^{i}(-1)^{n+1-i} P_{i c} 2^{i}-\sum_{i=0}^{n} C_{n}^{i}(-1)^{n-i} P_{i c} 2^{i} \\
& \quad=3^{n} e^{-\alpha} \sum_{i=0}^{n+1} C_{n+1}^{i}(-1)^{n+1-i} P_{i c} 2^{i}-3^{n+1} e^{-\alpha} \sum_{i=0}^{n} C_{n}^{i}(-1)^{n-i} P_{i c} 2^{i}, \\
& \left(3^{n} \sum_{i=0}^{n+1} C_{n+1}^{i}(-1)^{n+1-i} P_{i c} 2^{i}-3^{n+1} \sum_{i=0}^{n} C_{n}^{i}(-1)^{n-i} P_{i c} 2^{i}\right) e^{-\alpha} \\
& \quad=\sum_{i=0}^{n+1} C_{n+1}^{i}(-1)^{n+1-i} P_{i c} 2^{i}-\sum_{i=0}^{n} C_{n}^{i}(-1)^{n-i} P_{i c} 2^{i} .
\end{aligned}
$$

It follows from $\operatorname{deg} \alpha=1$ that

$$
\left\{\begin{array}{l}
3^{n+1} \sum_{i=0}^{n} C_{n}^{i}(-1)^{n-i} P_{i c} 2^{i}-3^{n} \sum_{i=0}^{n+1} C_{n+1}^{i}(-1)^{n+1-i} P_{i c} 2^{i} \equiv 0 \\
\sum_{i=0}^{n} C_{n}^{i}(-1)^{n-i} P_{i c} 2^{i}-\sum_{i=0}^{n+1} C_{n+1}^{i}(-1)^{n+1-i} P_{i c} 2^{i} \equiv 0
\end{array}\right.
$$

Then, by (3.19),

$$
3 \sum_{i=0}^{n} C_{n}^{i}(-1)^{n-i} P_{i c} 2^{i}=\sum_{i=0}^{n+1} C_{n+1}^{i}(-1)^{n+1-i} P_{i c} 2^{i},
$$


and

$$
\sum_{i=0}^{n} C_{n}^{i}(-1)^{n-i} P_{i c} 2^{i}=\sum_{i=0}^{n+1} C_{n+1}^{i}(-1)^{n+1-i} P_{i c} 2^{i} \equiv 0
$$

By our assumption, $P=s z^{n-t}+P^{*}$, where $s \neq 0$ and $\operatorname{deg} P^{*}<n-t$. Then the coefficient of $z^{n-t}$ of $(3.20)$ is

$$
s \sum_{i=0}^{n+1} C_{n+1}^{i}(-1)^{n+1-i} 2^{i} \equiv 0 .
$$

It follows that

$$
s=0,
$$

a contradiction.

Case 2.2.3. $\operatorname{deg} \alpha=0$, then $\alpha$ is a constant.

If $e^{\alpha}=1$, then $e^{\beta}=1$. By (3.1), we have

$$
f \equiv \Delta_{c}^{n} f \equiv \Delta_{c}^{n+1} f
$$

then $\Delta_{c} f \equiv \Delta_{c}^{n+1} f$, hence $f \equiv \Delta_{c} f$.

If $e^{\alpha} \neq 1$, by (3.13), we have

$$
F=-e^{-\alpha} P,
$$

thus

$$
f=A P(z),
$$

where $P(z)$ is a polynomial with $\operatorname{deg} P(z) \leq n-1$, and $A=1-e^{-\alpha}(\neq 0,1)$. But it contradicts the assumption that $f(z)$ is a transcendental function.

Thus, Theorem 8 is proved.

Acknowledgements

The authors thank the referee for his/her valuable suggestions to improve the present article.

\section{Funding}

Research was supported by the NNSF of China (Grant No. 11701188; 11371149).

\section{Competing interests}

The authors declare that they have no competing interests.

Authors' contributions

All the authors drafted the manuscript, and read and approved the final manuscript

\section{Publisher's Note}

Springer Nature remains neutral with regard to jurisdictional claims in published maps and institutional affiliations. 
References

1. Bergweiler, W., Langley, J.K.: Zeros of differences of meromorphic functions. Math. Proc. Camb. Philos. Soc. 142(1), 133-147 (2007)

2. Chang, J.M., Fang, M.L.: Entire functions that share a small function with their derivatives. Complex Var. Theory Appl. 49(12), 871-895 (2004)

3. Chen, B.Q., Chen, Z.X., Li, S.: Uniqueness theorems on entire functions and their difference operators or shifts. Abstr. Appl. Anal. 2012, Article ID 906893 (2012)

4. Chen, B.Q., Li, S.: Uniqueness problems on entire functions that share a small function with their difference operators. Adv. Differ. Equ. 2014, 311 (2014)

5. Chiang, Y.M., Feng, S.J.: On the Nevanlinna characteristic of $f(z+\eta)$ and difference equations in the complex plane. Ramanujan J. 16(1), 105-129 (2008)

6. Deng, B.M., Liu, D., Fang, M.L.: Unicity of meromorphic functions and their difference operators. Adv. Differ. Equ. 2018, $194(2018)$

7. El Farissi, A., Latreuch, Z., Asiri, A.: On the uniqueness theory of entire functions and their difference operators Complex Anal. Oper. Theory 10, 1317-1327 (2016)

8. El Farissi, A., Latreuch, Z., Belaïdi, B., Asiri, A.: Entire functions that share a small function with their difference operators. Electron. J. Differ. Equ. 2016, 32 (2016)

9. Halburd, R.G., Korhonen, R.J.: Difference analogue of the lemma on the logaritheoremic derivative with applications to difference equations. J. Math. Anal. Appl. 314(2), 477-487 (2006)

10. Halburd, R.G., Korhonen, R.J.: Nevanlinna theory for the difference operator. Ann. Acad. Sci. Fenn., Math. 31(2), 463-478 (2006)

11. Hayman, W.K.: Meromorphic Functions. Oxford Mathematical Monographs. Clarendon, Oxford (1964)

12. Jank, G., Mues, E., Volkmann, L.: Meromorphe Funktionen, die mit ihrer ersten und zweiten Ableitung einen endlichen Wert teilen. Complex Var. Theory Appl. 6(1), 51-71 (1986)

13. Latreuch, Z., El Farissi, A., Belaïdi, B.: Entire functions sharing small functions with their difference operators. Electron. J. Differ. Equ. 2015, 132 (2015)

14. Li, P., Yang, C.C.: Uniqueness theorems on entire functions and their derivatives. J. Math. Anal. Appl. 253(1), 50-57 (2001)

15. Yang, C.C., Yi, H.X.: Uniqueness Theory of Meromorphic Functions. Mathematics and Its Applications, vol. 557. Kluwer Academic, Dordrecht (2003)

16. Yang, L.: Value Distribution Theory. Springer, Berlin (1993)

\section{Submit your manuscript to a SpringerOpen ${ }^{\circ}$ journal and benefit from:}

- Convenient online submission

- Rigorous peer review

- Open access: articles freely available online

- High visibility within the field

- Retaining the copyright to your article

Submit your next manuscript at $>$ springeropen.com 